\section{The 2010 NCCN Clinical Practice Guidelines in Oncology on Prostate Cancer}

This issue includes the latest version of the NCCN Clinical Practice Guidelines in Oncology on Prostate Cancer, as well as several articles that clarify and expand on themes in those guidelines.

For example, the excellent manuscript from Lee and Amling provides the basis for updating the best available nomograms in the 2010 version of the NCCN Guidelines to help provide more individualized recommendations. This need for individualization includes both choice of treatment (active surveillance vs. radical prostatectomy vs. external beam radiation vs. brachytherapy) and type of treatment (need for neurovascular bundle resection and/or pelvic lymph node dissection during radical prostatectomy) for clinically localized prostate cancer or adjuvant or salvage radiation therapy for biochemical recurrence after radical prostatectomy.

The review by Nielson, Trock, and Walsh describes the evolution of the Johns Hopkins' position on salvage or adjuvant radiation therapy. Reanalysis of this experience led to a change from a relatively rare recommendation of additional treatment to the recognition that use of the "Johns Hopkins criteria" for local versus systemic recurrence was denying radiation to many men who might benefit.

Finally, Saylor and Smith provide a detailed description of the morbidity associated with androgen deprivation therapy and recommend evidence-based steps that urologists, radiation urologists, and medical oncologists should take in collaboration with primary care physicians to minimize the impact of androgen deprivation therapy on quality of life.

These articles expand on 3 of the many changes in the 2010 NCCN Guidelines on Prostate Cancer. Perhaps the most significant change is the recommendation for active surveillance and only active surveillance for men with "low risk" prostate cancer who have a life expectancy less than 10 years. In addition, a new "very low risk" category was created using a modification of the Epstein criteria for clinically insignificant prostate cancer wherein active surveillance is the only recommended option for men whose life expectancy is less than 20 years. These recommendations grow from 2 fundamental factors appreciated by the guideline panel.

First, growing evidence suggests that overtreatment of prostate cancer subjects too many men to side effects that outweigh a very small risk of prostate cancer death. This issue was discussed by Klotz ${ }^{1}$ in an earlier issue of JNCCN and led to the first major modification of the NCCN Guidelines on Prostate Cancer regarding active surveillance. Second, the continued maturation of experience by many medical centers with large series of men on active surveillance allows better recognition of men for whom risk of death from prostate is very low.

The 2010 NCCN Guidelines on Prostate Cancer have been revised substantially. As a consequence, the discussion also has been updated with great care. The Panel recognizes the difficulty of predicting the future of individual patients, but the members feel strongly that continued treatment of all men in whom prostate cancer can be diagnosed is as wrong as denying treatment to every man in whom prostate cancer is diagnosed. Best practice lies between these extremes, and the Panel hopes that both physicians who treat men with prostate cancer and the men and their loved ones who confront a prostate cancer diagnosis will find the 2010 NCCN Clinical Practice Guidelines in Oncology on Prostate Cancer useful.

\section{Reference}

1. Klotz L. Point: Active surveillance for favorable risk prostate cancer. J Natl Compr Canc Netw 2007;5:693-698.

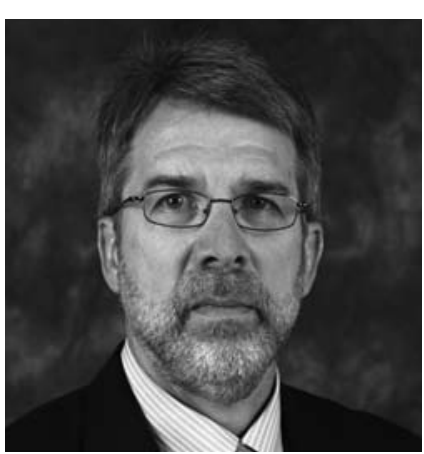

James L. Mohler, MD

Dr. Mohler is Associate Director for Translational Research and Senior Vice President, Chair of the Department of Urology, Founder of the Prostate Program, and Professor of Oncology at Roswell Park Cancer Institute. $\mathrm{He}$ is also Professor of Urology at the University at Buffalo School of Medicine and Biomedical Sciences and maintains academic appointments at UNC-Lineberger Comprehensive Cancer Center.

$\mathrm{He}$ is a Diplomate of the National Board of Medical Examiners and American Board of Urology and a Fellow of the American College of Surgeons. Dr. Mohler is Chair of the NCCN Prostate Cancer Guidelines Panel, Vice-Chair of the Urology Committee of the Cancer and Leukemia Group B, and Past-President of the Society for Basic Urologic Research. His professional society memberships include AMA, American Urological Association, and American College of Surgeons. Dr. Mohler's clinical practice focuses up prostate cancer and robot-assisted laparoscopic surgery. His laboratory focuses on the role of the androgen receptor in racial differences in prostate cancer aggressiveness and recurrence during androgen deprivation therapy. He has authored or co-authored more than 150 publications, including a book on prostate cancer. He serves on several journal editorial boards, including The Prostate and JNCCN, and reviews for several more, including Cancer and Clinical Cancer Research.

The ideas and viewpoints expressed in this commentary are those of the author and do not necessarily represent any policy, position, or program of the NCCN. 